\title{
Flow Characteristics of Two-Dimensional Supersonic Under-Expanded Coanda-Reattached Jet
}

\author{
Tetsuji Ohmura',2, Toshihiko Shakouchi'1, Shunsuke Fukushima1', Koichi Tsujimoto ${ }^{1}$ \\ ${ }^{1}$ Graduate School of Engineering, Mie University, Tsu, Japan \\ ${ }^{2}$ Fukuda Metal Foil \& Powder Co., Ltd., Kyoto, Japan \\ Email: shako@mach.mie-u.ac.jp
}

How to cite this paper: Ohmura, T., Shakouchi, T., Fukushima, S. and Tsujimoto, K. (2021) Flow Characteristics of TwoDimensional Supersonic Under-Expanded Coanda-Reattached Jet. Journal of Flow Control, Measurement \& Visualization, 9, 1-14.

https://doi.org/10.4236/jfcmv.2021.91001

Received: July 22, 2020

Accepted: October 22, 2020

Published: December 9, 2020

Copyright () 2021 by author(s) and Scientific Research Publishing Inc. This work is licensed under the Creative Commons Attribution International License (CC BY 4.0)

http://creativecommons.org/licenses/by/4.0/

\section{(c) (i) Open Access}

\begin{abstract}
When there is a wall near the jet, it deflects and flows while being attached to the wall owing to the Coanda effect. The flow characteristics of the incompressible and two-imensional (2D) Coanda-reattached jets have been considerably explained. However, 2D supersonic under-expanded jets, reattached to side walls, have not been sufficiently investigated. These jets are used in gas-atomization to produce fine metal powder particles of several micrometers to several tens micrometers. In this case, the supersonic under-expanded jets are issued from an annular nozzle, which is set around a vertically installed circular nozzle for molten metal. The jet flow at the center cross-section of the annular jet resembles a 2D Coanda-reattached jet that deflects and attaches on the central axis. In this study, the flow characteristics of a supersonic under-expanded Coanda air jet from a 2D nozzle that reattaches to an offset side wall are elucidated through experiment and numerical analysis. For numerical analysis, we show how much it can express experimental results. The effects of supply pressure $P_{0}$ on the flow characteristics such as the flow pattern, size of shock cell, reattachment distance, and velocity and pressure distributions, etc. are examined. The flow pattern was visualized by Schlieren method and the velocity distribution was measured using a Pitot tube. These results will be also useful in understanding the flow characteristics of a gas-atomization annular nozzle approximately.
\end{abstract}

\section{Keywords}

Supersonic Flow, Under-Expanded Jet, Coanda Reattached Jet, Flow Visualization, Gas-Atomization, Experimental Analysis, Numerical Analysis 


\section{Introduction}

When there is a wall near the jet, the jet will adhere to and flow round nearby the wall. This Coanda-reattached flow is used for controlling flow in a wall attached type fluidic device, preventing flow separation, and increasing the wing lift force. The flow characteristics of incompressible two-dimensional (2D) Coanda-reattached jet flows have been elucidated considerably owing to the development of fluidics [1] [2]. For example, Bourque et al. [1] studied the reattachment distance and pressure within the separation bubble of a $2 \mathrm{D}$ reattached jet by a theoretical analysis using momentum theory, and compared them with experiments in terms of the offset distance and inclined angle of the side wall.

Supersonic under-expanded free jets, such as jets from jet engines and rocket engines to obtain thrust have been studied in detail. Donaldson et al. [3] classified the flow patterns of sub- and supersonic under-expanded jets into three, depending on the pressure ratio of the supply pressure to the ambient pressure. Kojima et al. [4], Zapryagaev et al. [5], and André et al. [6] studied the structure of shock waves, the pressure and velocity distribution of supersonic under-expanded jets. Shadow et al. [7] and Zaman [8] showed the compressible spreading rates and mixing characteristics of supersonic coaxial jets. Katanoda et al. [9] [10] explained the effects of the nozzle divergence angle and Mach number on the shock cell length, and Sugawara et al. [11] showed the structure of three-dimensional microjet with a Mach disc by Mach-Zender in interferometer. Zaman [8] examined the flow characteristics of sub- and supersonic jets from special nozzles, whereas Shakouchi [12] studied those from an orifice nozzle. Franquet et al. [13] reviewed the studies on free under-expanded jets in a quiescent medium. However, studies regarding supersonic under-expanded Coanda-reattached jet flows and their flow characteristics are rare [14] [15].

For example, supersonic under-expanded jets are used in gas-atomization, producing fine metal particles of several micrometers to several tens of micrometers [16] [17] [18]. The molten metal from a circular nozzle is broken, subdivided, and refined by a shearing force caused by the large velocity gradient outside the supersonic under-expanded jet issued from a coaxial annular nozzle installed near the circular nozzle, and high frequency fluctuations caused by the flow (Figure 1). The atomized molten metal, which is spherical owing to surface tension, is subsequently cooled to produce fine metal powder particles. In this case, the supersonic under-expanded jet from the annular nozzle curves inward, deflects, and flows over the cone shape collectively because the pressure inside the annular jet decreases owing to jet entrainment, and the jet flow at the center section of the $x-y$ plane of the annular jet resembles a 2D Coanda-reattached jet that deflects and attaches on the central axis. To improve the production efficiency of the gas-atomization and control the particle size and distribution, the flow characteristics of the supersonic under-expanded Coanda-reattached jet from an annular nozzle must be studied. 


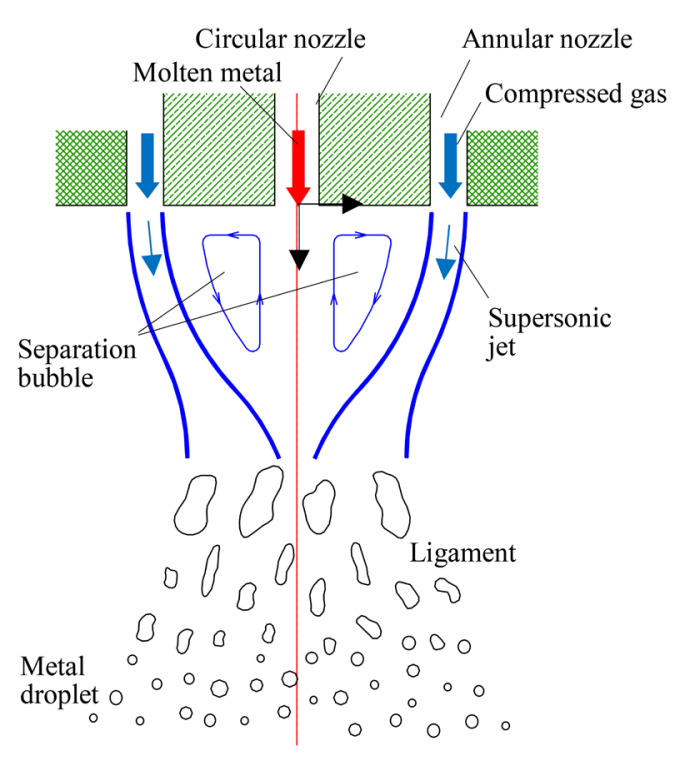

Figure 1. Gas-atomization nozzle and flow pattern.

In this study, the flow characteristics of a supersonic under-expanded Coanda air jet from a 2D nozzle that reattaches to an offset side wall are elucidated through experimental and numerical analyses. For numerical analysis, we show how much it can express experimental results. The effects of supply pressure $P_{0}$ on the flow characteristics such as the flow pattern, size of shock cell, reattachment distance, and velocity and pressure distributions are examined. The flow pattern was visualized using the Schlieren method and the velocity distribution was measured using a Pitot tube. $P_{0}$ was changed between $0.2 \mathrm{MPa}$ to $0.5 \mathrm{MPa}$. These results will also be useful in understanding the flow characteristics of a gas-atomization annular nozzle approximately.

\section{Flow Model}

Figure 2 shows the flow model and an illustration of how the 2D air jet issued from the nozzle of width $b$ reattaches to the side wall with an offset distance $D$ at the reattaching distance $x_{\mathrm{R}}$ while surrounding a separation bubble region of pressure $P_{\mathrm{b}}$. In this case, the jet centerline can be approximated by a circular arc of radius $R$ with its origin on the $y$-axis [1].

\section{Numerical Analysis}

The CFD program, SOLIDWORKS Flow Simulation, was used for the numerical analysis. The governing equations obtained using the $k-\mathcal{E}$ turbulence model, and the Favre-averaged Navier-Stokes equation were analyzed based on the finite sedimentation and participation methods.

Figure 3 shows the schematic of the calculation model and the calculation mesh. An orthogonal coordinate system was used, and the control volume was a rectangular parallelepiped. The cells in the vicinity of the shape boundary, domain region, narrow nozzle passage, center of the jet, and the entrainment re- 
gion of the jet are subdivided into different levels. The cells are arbitrarily cut by the shape boundary, and one parallel six-specimen cell has a plurality of different control volumes, such as one solid and one fluid. In addition, the nozzle width, the length of the parallel section of the nozzle, and offset distance were $b$, $L_{\mathrm{w}} / b=4.0$, and $D / b=5.0$, respectively. The upper left of the flow channel contained an opening, for the molten metal for gas-atomization, but it was closed and calculated in this study. This was assumed not to affect the flow characteristics of the reattached jet. Calculations were performed primarily for the $2 \mathrm{D}$ channel and the channel with a nozzle $A R=4.0$. The supply boundary entrance condition, that is, the supply air setting, was set to $P_{0}=0.2-0.5 \mathrm{MPa}$ at the static pressure setting. The exit boundary condition was set to atmospheric pressure. After investigated the effects of the number of cells on the flow, $1.13 \times 10^{6}$ cells were used.

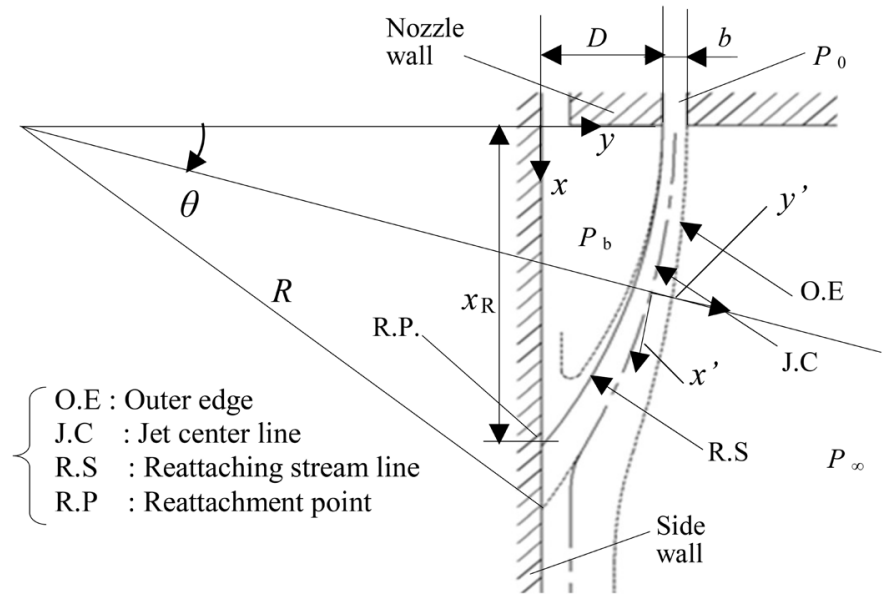

Figure 2. Flow model of reattached jet.

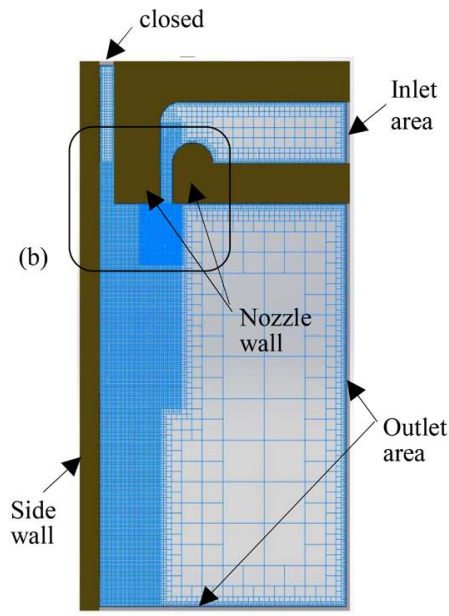

(a) Discretization mesh with boundary conditions

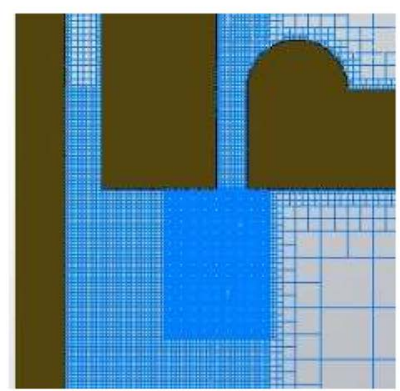

(b) Different level of mesh refinements

Figure 3. Computational domain and discretization mesh for two-dimensional numerical calculation (nozzle width: $b$, offset distance: $D / b=5.0$ ). 


\section{Two-Dimensional Jet}

Figure 4(a) shows the density distribution $\rho / \rho_{0}$ from numerical analysis, on the $x-y$ plane of the supersonic under-expanded reattached jet issued from the $2 \mathrm{D}$ nozzle of width $b$. The offset distance is $D / b=5.0$ and the supply pressure is $P_{0}=$ $0.4 \mathrm{MPa}$. In Figure 4(a), the length $C_{\mathrm{L}}$ and width $C_{\mathrm{W}}$ of the $1^{\text {st }}$ shock cell are also shown. Their details will be shown in the section 6.2. The jet from the $2 \mathrm{D}$ nozzle expands and compresses to form shock cells while generating shock waves and then flows away. In this case, owing to flow entrainment, the pressure on the side wall of the supersonic under-expanded jet decreases, and the jet is deflected and reattached to the side wall and flows down while forming a separation bubble. The reattaching distance $x_{\mathrm{R}}$, i.e., the value of $x$ at the reattachment point (Figure 2), also known as the reattaching distance, is $x_{\mathrm{R}} / b=16.1$. Furthermore, the minimum pressure and the position on the side wall are $P_{\mathrm{wmin}} / P_{0}=-0.046$, and $x / b=8.5$, respectively. In addition, the jet centerline can be expressed by an arc of radius $R / b=35.0$.

Figure 4(b) shows the density distribution $\rho / \rho_{0}$, from numerical analysis, of the $x-y$ plane at the center height of the flow channel of the supersonic under-expanded reattached air jet issued from a rectangular nozzle with aspect ratio $A R=H / b=4.0$ ( $H$ : nozzle height). The flow patterns in Figure 4(a) and Figure $4(\mathrm{~b})$ are approximately the same, and the reattaching distance $X_{\mathrm{R}}$, minimum bubble pressure $P_{\mathrm{b}}$, and radius $R$ are also approximately the same; $x_{\mathrm{R}} / b=$ $16.1,15.8, P_{\text {wmin }}=-0.046,-0.046$, and $R / b=35.0,36.5$, respectively. Consequently, it may be assumed that the flow state in the $x-y$ section at the center height of the flow channel with $A R=4.0$ in Figure 4(b) is two-dimensional. The experiments are carried out using the flow channel of $A R=4.0$, as shown in Figure 5.

\section{Experimental Apparatus and Procedure}

Figure 5 illustrates the experimental apparatus. The elements comprise brass nozzle walls, a side wall, and transparent upper and lower glass end plates. The air from the compressor passes through the reservoir, dryer, and flow meter (Azbil, air flow monitor, CMG500) and flows in the element; subsequently, it flows from the rectangular nozzle of width $b=2.5 \mathrm{~mm}(A R=4.0)$ and parallel length $L_{\mathrm{w}} / b=4.0$ to the ambient. In this case, a equal amount of compressed air was introduced through the $\varphi=8.5 \mathrm{~mm}$ inflow holes provided on the upper and lower end plates of the element. The jet reattaches to the side wall at an offset distance of $D / b=5.0$ by the Coanda effect and flows while forming a circulating separation bubble region between the jet and the side wall.

A static pressure hole, $\varphi=0.8 \mathrm{~mm}$, was provided at the center height of the channel before the nozzle, which was used to measure the supply pressure $P_{0}$. The supply pressure varied to a maximum of $P_{0}=0.5 \mathrm{MPa}$; subsequently, flow visualization and velocity and pressure measurements were performed.

A Schlieren optical system (Kato Koken, Japan, SS150) was used for flow visualization. A total or static Pitot tube with a diameter of $1.0 \mathrm{~mm}$ was used to 


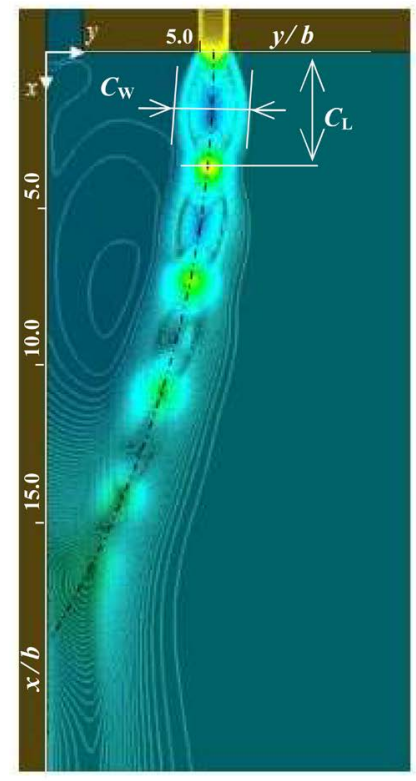

(a) Two-dimensional jet (2D)

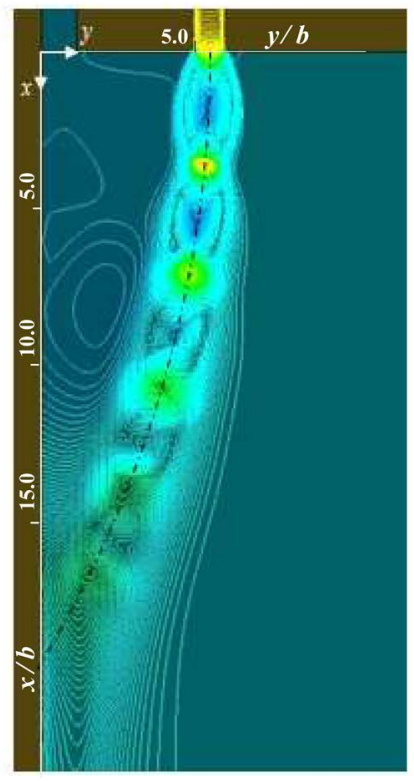

(b) $A R=H / b=4.0$

Figure 4. Flow pattern of supersonic under-expanded Coanda reattached jet (density distribution by numerical analysis, $P_{0}=0.4 \mathrm{MPa}$, nozzle width: $b$, offset distance of side wall: $D / b=5.0)$.

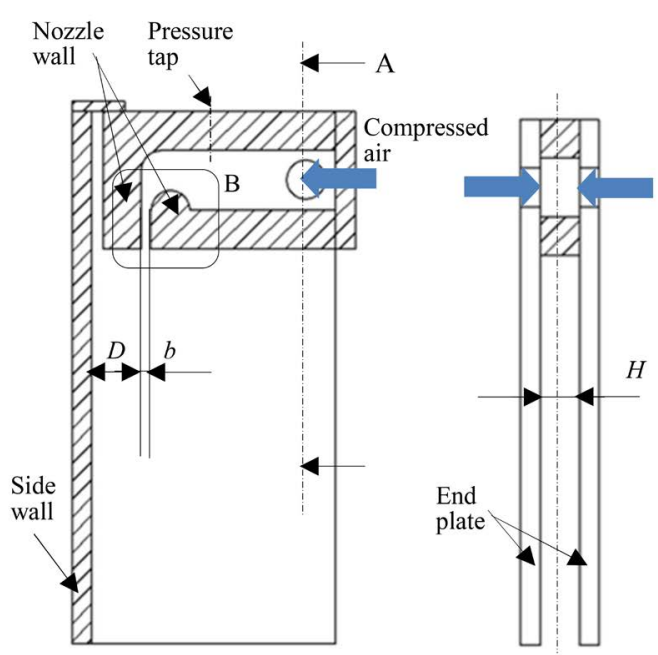

(a) Schematic diagram

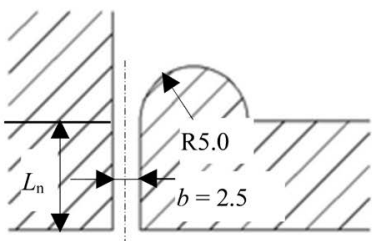

(b) Details of B

Figure 5. Experimental apparatus, element (nozzle width: $b=2.5 \mathrm{~mm}$, offset distance: $D / b=5.0$, height and nozzle aspect ratio: $A R(=H / b)=4.0)$.

measure the velocity at the center height of the element. The velocity distribution was obtained by the total and static pressure measurements while considering the compressibility. The Bourdon tube and mercury column manometers were used for pressure measurements. A three-dimensional (3D) traverse device was used to move the Pitot tube. The pressure distribution on the side wall was measured through pressure holes of $\varphi=0.8 \mathrm{~mm}$, installed every $3.0 \mathrm{~mm}$ in the $x$-direction at the center height of the side wall. The reattachment point of the jet 
was obtained using the tuft method [1]. Since the flow direction on the side wall changes back and forth with reattachment point as the boundary (Figure 2), the reattachment point can be estimated from the direction of a small tuft or thin thread slowly moved on the wall.

\section{Results and Discussions}

\subsection{Flow Patterns}

Figure 6(a)(1) shows the visualized Schlieren image of the supersonic reattached jet with $b=2.5 \mathrm{~mm}(A R=4.0), D / b=5.0$, and $P_{0}=0.2 \mathrm{MPa}$. The jet issued from the nozzle expanded in the $y$-direction and then compressed; subsequently, the shock cell was formed in the under-expanded jet. The white and dark colored areas in the shock cell approximately correspond to the expansion and compression regions, respectively. The jet forms a group of shock cells and then curves and reattaches to the side wall by the Coanda effect. The chain-line in the Figure shows the jet centerline with the origin on the $y$-axis when the jet center was approximated using a circular arc. Bourque et al. [1] showed that the centerline of a $2 \mathrm{D}$ incompressible reattached jet can be approximated by a circular arc. It is assumed that the jet centerline of the supersonic under-expanded reattached jet can also be approximated using a circular arc.

Figures 6(a)(2)-(4) show the results for $P_{0}=0.3-0.5 \mathrm{MPa}$, respectively. The length $C_{\mathrm{L}}$ and width $C_{\mathrm{W}}$ [Figure 6(b)(3)] of the shock cell increases with $P_{0}$, radius $R$ of the circular arc, and the reattachment distance $x_{\mathrm{R}}$. Figures $6(\mathrm{~b})(1)-(4)$ show the density distributions $\rho / \rho_{0}$ (from numerical analysis) for $P_{0}=0.2-0.5$ $\mathrm{MPa}$, respectively. Like the density distribution, the flow pattern, $C_{\mathrm{L}}, C_{\mathrm{W}}, R$, and $X_{\mathrm{R}}$ agree with the experimental results (details will be described later). That is, the flow characteristics of the supersonic under-expanded reattached jet for the flow passage with $A R=4.0$ are approximately represented by the numerical analysis.

\subsection{Shock Cell Size}

The jet issued from the nozzle formed a shock cell after expansion and compression; thereafter, while expansion and compression continued, it curved and reattached to the side wall and flowed downstream. Figure 7 shows the size, length $C_{\mathrm{L}}$, and maximum width $C_{\mathrm{W}}$ of the first shock cell (Figure 4(a)). The abscissa is $P_{0}$ and $P_{0 \mathrm{a}} / P_{\mathrm{aa}}$, where $P_{0 \mathrm{a}}$ and $P_{\mathrm{aa}}$ are supply and ambient pressures expressed in the absolute pressure, respectively. The $C_{\mathrm{L}}$ was obtained as the distance from the nozzle exit to the position where the measured jet centerline velocity is initially minimal (Figure 11). The $C_{\mathrm{w}}$ was obtained from the visualized flow pattern and the density distribution. At that time, the jet boundary was set at the position where the shade suddenly changed in the jet width direction. Both $C_{\mathrm{L}}$ and $C_{\mathrm{W}}$ increase almost linearly with $P_{0}$. The numerical analysis is consistent with the experimental results. In addition, the sizes of the second and third shock cells were similar to the first one. 

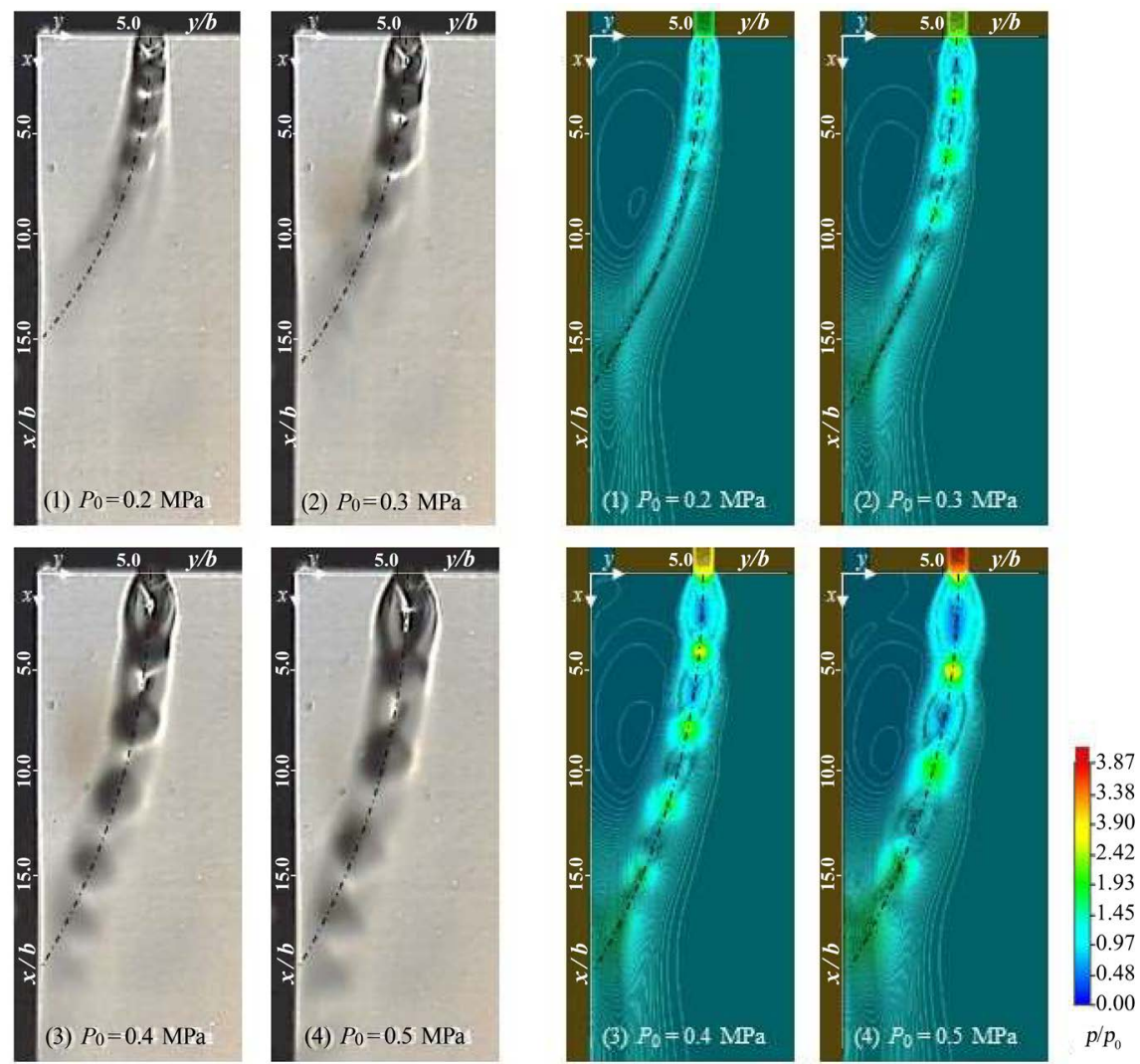

(a) Schlieren method

(b) Density distribution (Numerical analysis)

Figure 6. Visualized flow pattern.

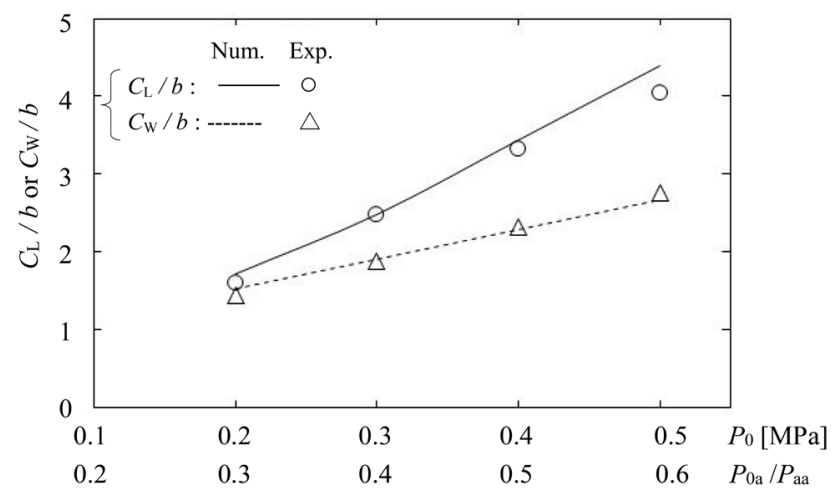

Figure 7. Length $C_{L}$ and width $C_{W}$ of the 1 st shock cell.

\subsection{Centerline of the Jet and Reattached Distance}

As mentioned in Section 5.1, the jet centerline can be represented by a circular arc.

Figure 8 shows the radius $R$ of a circular arc, along with the numerical analysis. The centerlines for the numerical analysis and the experimental result when $P_{0}=0.4 \mathrm{MPa}$ were obtained using the least-squares method by specifying the position of the maximum velocity from the velocity distribution of each jet cross-section. The $R$ increases with $P_{0}$ and is almost constant at $R / b=34.6$ after 
reaching the maximum at $P_{0}=0.4 \mathrm{MPa}$. Thus, in a supersonic under-expanded reattached jet, the centerline can be approximated by a circular arc. Furthermore, the numerical analysis of $R$ and $x_{\mathrm{R}}$ reflects the experimental results accurately for $P_{0} \geq 0.4 \mathrm{MPa}$. The reattachment distance $x_{\mathrm{R}} / b$, shown in Figure 8 increases with $P_{0}$ and then remains constant at 16.1. However, the numerical calculations for $P_{0}=0.2,0.3$ don't reflect the experimental results well.

\subsection{Pressure Distribution on Side Wall}

Figure 9 shows the pressure distribution $P_{\mathrm{w}} / P_{0}$ on the side wall for $P_{0}=0.2-0.5$ $\mathrm{MPa}$. The jet issued from the nozzle entrains the surrounding fluid owing to the large velocity gradient and the fluid viscosity. When a solid wall exists near the jet, it entrains and removes the fluid from the finite area between the jet and the wall. Consequently, the pressure decreases, and the jet deflects and reattaches, enclosing the circulating separation bubble to the side wall. The pressure $P_{\mathrm{w}} / P_{0}$ on the side wall has a negative value corresponding to the separation bubble; it decreases with increasing $x / b$ and reaches a minimum and approaches the ambient pressure. The minimum of the negative value and maximum of $P_{\mathrm{w}} / P_{0}$ increase with $P_{0}$, and are almost the same at $P_{0} \geq 0.4 \mathrm{MPa}$. This is because increasing the velocity owing to the increase in $P_{0}$ hinders the jet from entraining the surrounding fluid [12], jet flows penetrates. The numerical analysis reflected the experimental well, particularly when $P_{0} \geq 0.3 \mathrm{MPa}$, that is, when $P_{0}$ is large.

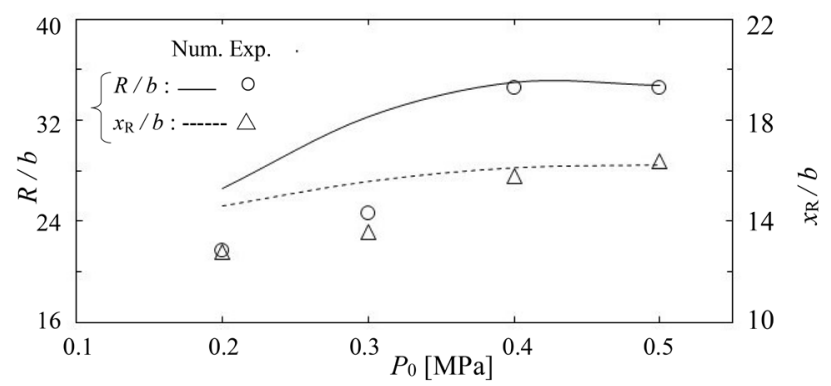

Figure 8. Radius $R$ of jet centerline and reattachment distance $x_{\mathrm{R}}$ of the reattaching jet.

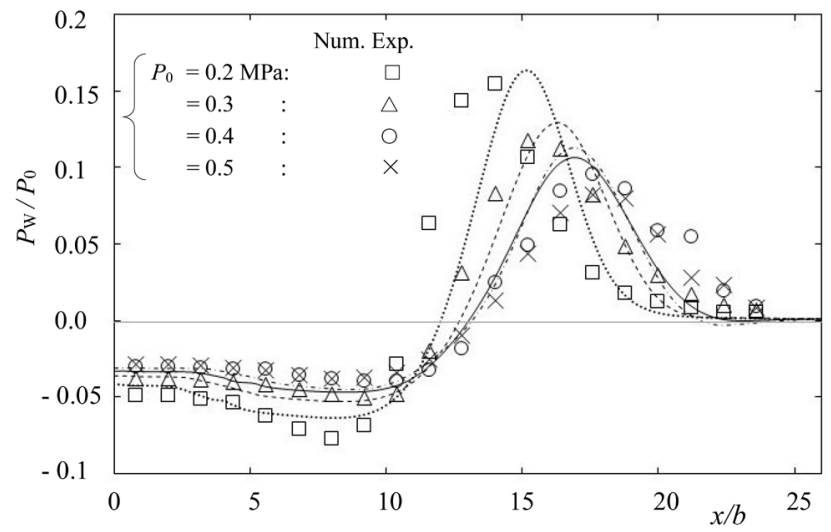

Figure 9. Surface pressure distribution $P_{\mathrm{w}} / P_{0}$ on the side wall. 
Figure 10 shows the minimum pressure $P_{\text {wmin }} / P_{0}$ on the side wall. $P_{\text {wmin }} / P_{0}$ decreases with increasing $P_{0}$ and approaches a constant at $P_{0} \geq 0.4 \mathrm{MPa}$, and the numerical analysis reflects the experiment effectively. In addition, according to Bourque et al. [1], the position $x$ of the minimum pressure on the side wall of a $2 \mathrm{D}$ incompressible reattached jet corresponds to the position $x$ of the minimum bubble pressure. This is reflected in the numerical analysis of the supersonic under-expanded reattached jet investigated in this study.

\subsection{Pressure Difference between Both Sides of Reattached Jet and Jet Circular Motion}

Bourque et al. [1] reported that when a $2 \mathrm{D}$ incompressible jet flow reattaches to a side wall set parallel to the nozzle axis at an offset distance, the jet centerline can be approximated to a circular arc of radius $R$ (Figure 2). The relationship between the pressure difference $\Delta P$ on both sides of the jet and the centrifugal force exerted on the jet can be expressed as follows, where the inside pressure of the reattached jet is the minimum pressure of the separation bubble.

$$
\Delta P=J / R \text {. }
$$

where $J$ is the jet momentum.

Equation (1) can be rewritten as

$$
\Delta P=\frac{\rho_{0} Q^{2}}{b H^{2} R} .
$$

where $Q$ and $\rho_{0}$ are the flow rate and density of air at standard conditions, respectively.

This relationship was investigated for a supersonic under-expanded reattached jet of $P_{0}=0.4 \mathrm{MPa}$, assuming that the jet momentum was conserved, as in Bourque et al. [1]. Here, because the minimum pressure on the side wall, flow rate, and arc radius were $P_{\text {wmin }}=1.58 \times 10^{4}[\mathrm{~Pa}], Q=58.0\left[\mathrm{Nm}^{3} / \mathrm{h}\right]$, and $R=86.5$ $\mathrm{mm}$, respectively. Inserting the jet momentum derived from $Q$ and $R$ into Equation (1) yields $\Delta P=1.55 \times 10^{4}[\mathrm{~Pa}]$. This is almost the same as $P_{\text {wmin }}$, which is reflected in the case of the supersonic reattached jet. Therefore, the relationship shown in Equation (1) holds well.

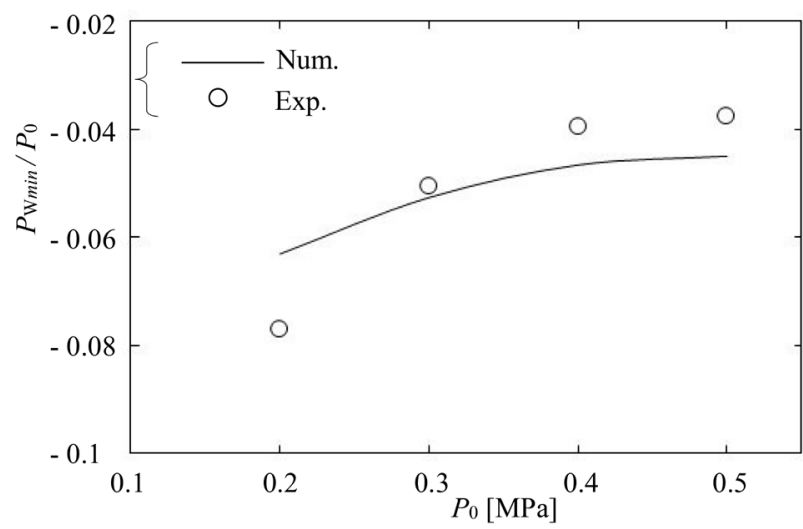

Figure 10. Minimum surface pressure $P_{\text {wmin }} / P_{0}$ on the side wall. 


\subsection{Centerline Velocity of Supersonic Reattached Jet}

Figure 11 shows the numerical calculations of the centerline velocity $u_{c}$ of the supersonic reattached jet. The abscissa is the angle $\theta$ (angle in the circumferential direction with nozzle exit set at $\theta=0^{\circ}$, Figure 2.) when the jet centerline is approximated by a circular arc. The case of experiment for $P_{0}=0.4 \mathrm{MPa}$ is also shown in Figure 11. Based on the numerical results for $P_{0}=0.4 \mathrm{MPa}$, the jet issues from the nozzle initially expanding and the $u_{\mathrm{c}}$ reaches a maximum at $\theta=$ $3.25^{\circ}$ and then decreases by compression to a minimum at $\theta=6.0^{\circ}$. It is assumed that the region from $\theta=0^{\circ}$ to $6.0^{\circ}$ corresponds to the first shock cell. Thereafter, the $u_{c}$ decays gradually while repeating expansion and compression. The amplitude of the increase/decrease in $u_{c}$ reduces, but the amplitude of $u_{c}$ is moderate. The same is applicable to the second and third shock cells, and their positions $\theta$ are almost the same based on numerical analysis and experiment. However, the behavior of velocity $u_{c}$ in the shock cells differs, and the values from the numerical analysis are larger and smaller than those of the experiments in the back and front halves of the shock cell, respectively. The numerical analysis for $P_{0}=0.4$ $\mathrm{MPa}$ expresses the experimental results qualitatively. However, it does not represent the reality of the experiments. In addition, the maximum of $u_{\mathrm{c}}$ and the length of the shock cell increased with $P_{0}$.

\subsection{Equi-Velocity Distribution and Velocity Distribution at Cross-Section}

Figure 12 shows an example of the equi-velocity diagram and flow direction for the case, $P_{0}=0.4 \mathrm{MPa}$. The jet from the nozzle expands and the velocity increases (red colored area), and then compresses and the velocity decreases. The jet flow decreases downstream, while expansion and compression are repeated. The jet reattaches to the side wall, and recirculation flow is in the separation bubble.

Figure 13 shows the experimental results of the velocity distribution $u$ at the cross-sections of (a): $\theta=3.25^{\circ}$ in the middle of the compression region; (b): $\theta=$ $6.5^{\circ}$ in the maximum compressed region of the first shock cell; and (c), (d): $\theta=$ $12.5^{\circ}$ and $18.25^{\circ}$ in the second and third most compressed regions (Figure 12), respectively. The $y^{\prime}$ on the vertical axis in Figure 13 is the distance in the radius direction from the centerline of the reattached jet (Figure 2). The cross-sectional velocity profile of a supersonic under-expanded jet differs depending on the expansion or compression regions. Kojima, T. et al. [4] showed that the velocity profile in the shock cell of a supersonic under-expanded free jet was concave initially, convex in the middle, and nipple-shaped at the end. The velocity profile at $\theta=3.25^{\circ}$ was concave and asymmetric, with a minimum at the jet center, which differs from Kojima's classification of convex. This could because this jet was a reattached jet, and the pressure on the separation bubble side was negative. The profiles at $\theta=6.5^{\circ}, 12.5^{\circ}$, and $18.25^{\circ}$ are convex at the most compressed the first shock cell is convex, that at $\theta=6.5^{\circ}$ (" 1 st Com.") is concave, and those at $\theta^{\circ}$ $=12.5^{\circ}, 18.25^{\circ}$ ("2nd and 3rd Com.") are convex. As shown by the jet centerline velocity in point of the local minimum. Additionally, numerical calculations are 


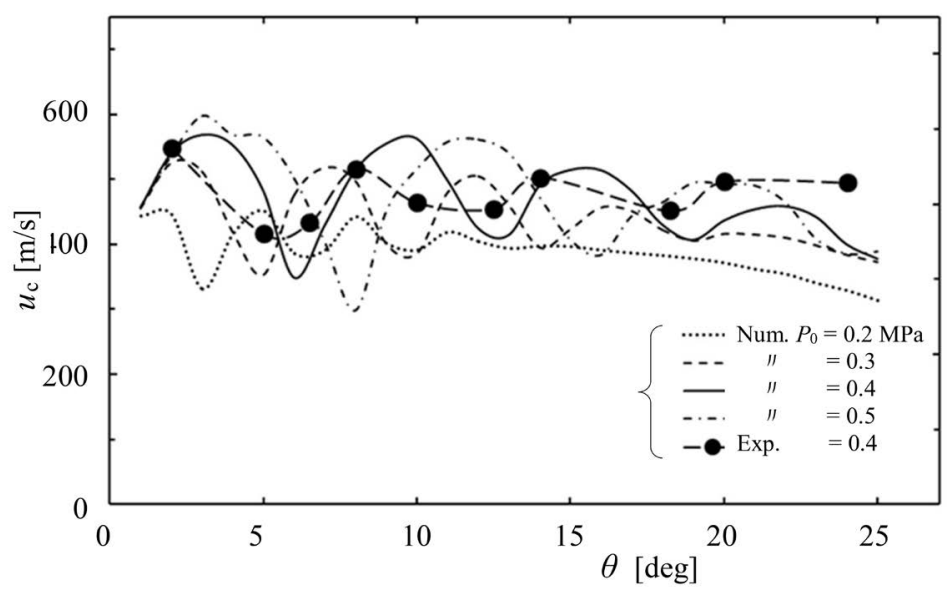

Figure 11. Jet centerline velocity $u_{c}$ of the reattached jet.

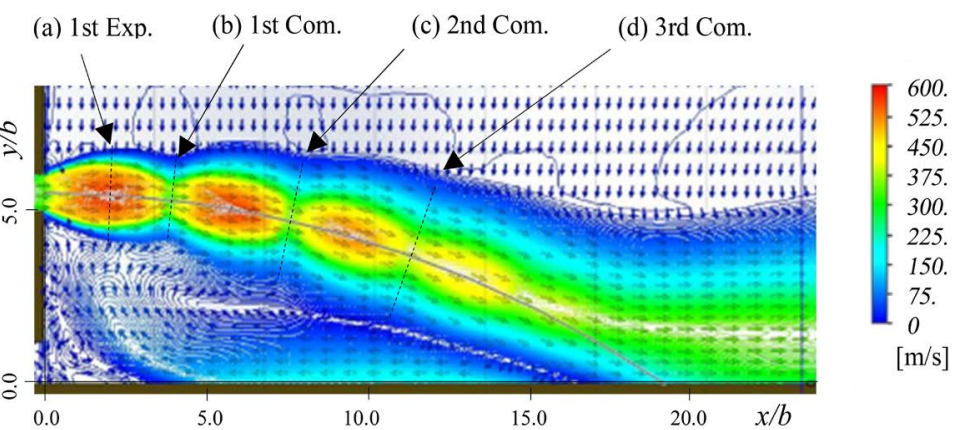

Figure 12. Equi-velocity distribution flow direction for $P_{0}=0.4 \mathrm{MPa}$ (numerical results).

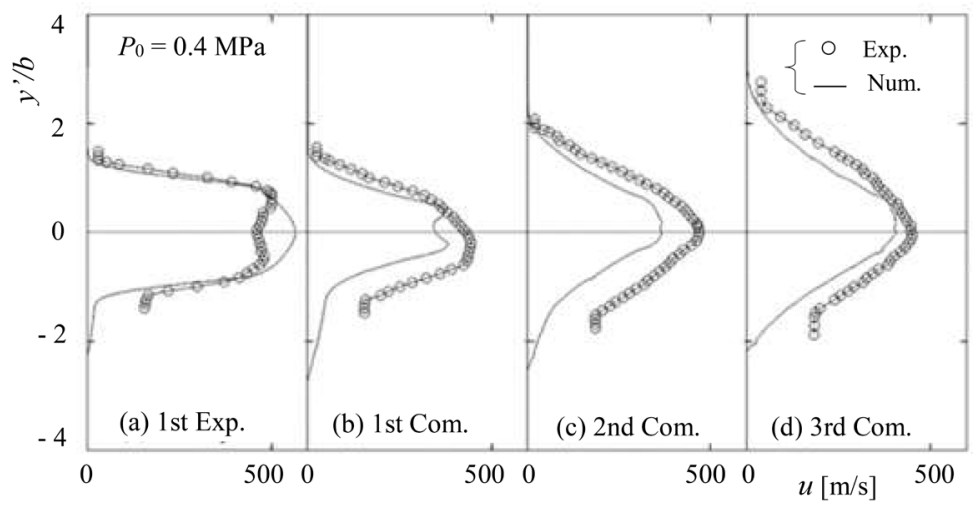

Figure 13. Velocity distribution at the cross-section for $P_{0}=0.4 \mathrm{MPa}$ (experimental and numerical results).

shown in Figure 13. The jet width and range of the numerical results are smaller than those of the experimental results, however, the numerical calculation reflects the approximate distribution of the experiment.

\section{Conclusions}

In this study, flow characteristics, such as shock cell formations, moving trajectory of reattached centerline approximated by a circular arc, reattachment dis- 
tance, pressure distribution on a side wall, jet centerline velocity, and velocity distribution at the cross-section of a $2 \mathrm{D}$ supersonic under-expanded jet reattached were studied through experiment and numerical analyses. For numerical analysis, we showed how much it could represent experimental results. The findings are as follows:

1) A supersonic under-expanded jet reattached to the side wall with an offset distance, surrounding the separation bubble region, as in a $2 \mathrm{D}$ incompressible reattached jet. Additionally, expansion and compression shock waves and shock cells were formed in this jet.

2) The centerline of a $2 D$ supersonic under-expanded reattached jet can be approximated by a circular arc of radius $R$, in which $R$ and the reattachment distance $x_{\mathrm{R}}$ increased with supply pressure $P_{0}$. Furthermore, the numerical calculations for supersonic reattached jet represent the experimental results fairly well.

The relationship $\Delta P=J / R$, obtained by assuming that the force owing to the pressure difference $\Delta P$ applied to both sides of the jet and the centrifugal force applied to the jet element were balanced, was applicable.

3) The length and width of the shock cell of the supersonic reattached jet increased with $P_{0}$, and the minimum pressure on the side wall decreased with increasing $P_{0}$. The numerical calculations represented the experimental outcomes well.

4) The jet centerline velocity fluctuated with its maximum and minimum values according to the expansion and compression of the jet, and the values increased with $P_{0}$. The numerical calculations for the supersonic reattached jet represented the experimental outcomes fairly well. The experimental and numerical results of the cross-sectional velocity distribution and jet width differed a little, however, the numerical calculations represented the experimental results fairly well.

5) The above results for the $2 \mathrm{D}$ reattached jet will also be useful in understanding the flow characteristics from a gas-atomization nozzle approximately.

\section{Conflicts of Interest}

The authors declare no conflicts of interest regarding the publication of this paper.

\section{References}

[1] Bourque, C. and Newman, B.G. (1960) Reattachment of a Two-Dimensional, Incompressible Jet to an Adjacent Flat Plate. Aeronautical Quarterly, 11, 201-232. https://doi.org/10.1017/S0001925900001797

[2] Kirshner, J.M. and Katz, S. (1975) Design Theory of Fluidic Components. Academic Press, Cambridge.

[3] Donaldson, C.D. and Snedeker, R.S. (1971) A Study of Free Jet Impingement, Part 1, Mean Properties of Free and Impinging Jets. Journal of Fluid Mechanics, 45, 281-319. https://doi.org/10.1017/S0022112071000053 
[4] Kojima, T. and Matsuoka, Y. (1986) Study on Underexpanded Sonic Jet (1st Report, on the Mechanism of Pseudo-Shock Waves and Pressure and Velocity in Jets). Transactions of the JSME, Ser. B, 52, 2021-2027. (In Japanese) https://doi.org/10.1299/kikaib.52.2021

[5] Zapryagaev, V.I., Kiselev, N.P. and Pivovarov, A.A. (2015) Gasdynamic Structure of an Axisymmetric Supersonic Underexpanded Jet. Fluid Dynamics, 50, 87-97. https://doi.org/10.1134/S001546281501010X

[6] André, B., Castelain, T. and Bailly, C. (2014) Experimental Exploration of Underexpanded Supersonic Jets. Shock Waves, 24, 21-32. https://link.springer.com/article/10.1007/s00193-013-0457-4 https://doi.org/10.1007/s00193-013-0457-4

[7] Shadow, K.C., Gutmark, E. and Wilson, K.J. (1990) Compressible Spreading Rates of Supersonic Coaxial Jets. Experiments in Fluids, 10, 161-167. https://doi.org/10.1007/BF00215025

[8] Zaman, K.B.M.Q. (1999) Spreading Characteristics Compressible Jets from Nozzles of Various Geometries. Journal of Fluid Mechanics, 383, 197-228. https://doi.org/10.1017/S0022112099003833

[9] Katanoda, H., Miyazato, Y. and Masuda, M. (1995) Numerical Calculation of Under-Expanded Axisymmetric Supersonic Jet (Effects of Nozzle Divergence Angle and Mach Number on Shock Cell Length). Transactions of the JSME, Ser. B, 61, 3236-3242. (In Japanese) https://doi.org/10.1299/kikaib.61.3236

[10] Katanoda, H., Miyazato, Y., Masuda, Y. and Matsuo, K. (2000) Pitot Pressures of Correctly-Expanded and Under-Expanded Free Jets from Axisymmetric Supersonic Nozzles. Shock Waves, 10, 95-101. https://doi.org/10.1007/s001930050182

[11] Sugawara, S., Nakano, S., Miyazato, Y. and Ishino, Y. (2020) Three-Dimensional Reconstruction of a Microjet with a Mach Disc by Mach-Zehnder Interferometer. Journal of Fluid Mechanics, 893, A25. https://doi.org/10.1017/jfm.2020.217

[12] Shakouchi, T., Tanoue, T., Tsujimoto, K. and Ando, T. (2019) Flow Characteristics of Sub- and Supersonic Jets Issued from Orifice and Notched Orifice Nozzles, and Application to Ejector. Journal of Fluid Science and Technology, JSME, 14, 1-12.

[13] Franquet, E., Perrier, V., Gibout, S. and Bruel, P. (2015) Free Under-Expanded Jets in a Quiescent Medium: A Review. Progress in Aerospace Science, 77, 25-53. https://doi.org/10.1016/j.paerosci.2015.06.006

[14] Matsuo, S., Setoguchi, T., Kudo, T. and Yu, S. (1998) Study on the Characteristics of Supersonic Coanda Jet. Journal of Thermal Science, 7, 165-175.

https://doi.org/10.1007/s11630-998-0012-2

[15] Hortensius, R., Dutton, J.C. and Elliot, G.S. (2017) Near Field of an Axisymmetric Under-Expanded Jet and an Adjacent Parallel Surface. AIAA Journal, 55, 2489-2501. https://doi.org/10.2514/1.J055515

[16] Nourl, J.M. and Whitelaw, J.H. (1996) Flow Characteristics of an Under Expanded Jet and Its Application to the Study of Droplet Breakup. Experiments in Fluids, 21, 243-247. https://doi.org/10.1007/BF00190673

[17] Lavernia, E.J. and Srivatsan, T.S. (2010) The Rapid Solidification Processing of Material: Science, Principles, Technology, Advances, and Applications. Journal of Material Science, 45, 287-325. https://doi.org/10.1007/s10853-009-3995-5

[18] Üral, A. (1990) Production of Rapidly Aluminum Alloy Powders by Gas Atomization and Their Applications. Powder Metallurgy, 33, 53-64.

https://doi.org/10.1179/pom.1990.33.1.53 